quelques moyens financiers, il est possible de venir à bout de cette entreprise. Les résultats atteints paieront largement, au sens propre comme au sens figuré, de tous ces efforts.

Dès à présent, il est nécessaire de songer à cette période d'après guerre où il faudra redonner une vive impulsion à l'organisation agricole de notre empire. Toutes nos richesses, même les moindres, devront être exploitées. Dans cette intention, nous avons pensé que le développement d'une petite race locale pourrait contribuer à cette ouvre tout en apportant aux états du Levant sous mandat français un peu d'hygiène et de bien-être.

Beyrouth, le 4 juin 1940.

Lyon, le 28 janvier 1941.

\title{
BIBLIOGRAPHIE
}

J. EDdé. Géographie de la Syrie et du Liban.

Latron. La vie rurale en Syrie et au Liban.

L. Pigoury. Les piroplasmoses bovines au Levant.

Dr Portet et Gamland. La faune momifiée de l'ancienne Egypte.

M. Saba. L'Inspection des viandes et des abattoirs on Syrie. Thèse, Alfort, 1936.

Sanson. Traité de Zootechnie.

Statistiques des douanes du port de Beyrouth.

\section{PRÉPARATION ET PROPRIÉTÉS DES ESTERS LACTIQUES}

\author{
par G. GÉNIN
}

Ingénieur chimiste E. P. C.

Au cours des diverses études que nous avons publiées ici même sur les applications des produits contenus dans le sérum du lait, nous avons été amenés à parler assez fréquemment des esters lactiques et nous avons insisté en particulier sur quelques fabrications à la base desquelles on peut utiliser ces esters. WHITtien et Rogers [1], OLIve [2] et Burton [3] ont également signalél'emploi de ces esters et ils ont décrit leur préparation en partant d'acide lactique fabriqué par fermentation du sérum. Cet acide, en effet, peut être transformé en différents mono et diesters dont certains présentent un intérêt assuré. Plus récemment, L. T. SмiтH et H. V. Claborn [4] ont également étudié, au Bureau of Dairy Industry du Ministère de l'Agriculture des Etats-Unis, la préparation d'esters lactiques déjà connus et de certains nouveaux, en utilisant directement le lactate de calcium ou le lactate de sodium bruts que l'on obtient par la fermentation du sérum. A certains points de vue, 
la méthọde utilisée par ces deux auteurs présente plusieurs nouveautés et nous passerons rapidement en revue leur recherche.

L'acide lactique possède deux groupes hydroxyles actifs; on peut, par conséquent, préparer, en partant de cet acide, trois différents types d'esters :

1. L'hydroxyle du groupe $\mathrm{COOH}$ peut réagir avec un alcool et former un ester de l'acide lactique répondant à la formule $\mathrm{CH}^{3} \mathrm{CH}$ OH COOR [5].

2. Pour obtenir un éther de la $2^{\mathrm{e}}$ eatégorie, on fait réagir le groupe alcool secondaire en position $\alpha$ restant dans l'éther de la $1^{\text {re }}$ catégorie, soit avec des chlorures d'acide [6], soit avec des anhydrides d'acide [7] pour obtenir un produit répondant à la formule générale :

$$
\begin{gathered}
\mathrm{O}-\mathrm{OCR} \\
\text { I } \\
\mathrm{CH}^{3}-\mathrm{CH}-\mathrm{COOR}
\end{gathered}
$$

Dans ce composé, on voit qu'on a fait réagir les deux groupes $\mathrm{OH}$ de l'acide lactique pour obtenir un $\alpha$-oxypropionate.

3. Enfin, pour obtenir un ester de la $3^{e}$ eatégorie, on fait réagir le groupe alcool secondaire de l'acide lactique avec de l'anhydride acétique et un chlorure d'acide [8] de façon à obtenir un produit répondant à la formule générale:

$$
\begin{gathered}
\mathrm{O}-\mathrm{OCR} \\
\mathrm{I} \mathrm{CH}^{3}-\mathrm{CH}-\mathrm{COOH}
\end{gathered}
$$

Ce composé peut être également préparé [9] par saponification partielle de l' $\alpha$-oxypropionate d'éthyle.

L'éthérification de l'hydroxyle du groupe $\mathrm{COOH}$ s'effectue facilement en faisant réagir l'acide lactique avee l'alcool, en particulier si on opère en présence d'une petite quantité d'acide sulfurique comme catalyseur. Le rendement en esters peut varier de $60 \%$ environ à près de $100 \%$, il dépend essentiellement de la méthode que l'on emploie pour éliminer l'eau qui se forme au cours de l'éthérification. Il est toutefois plus difficile d'obtenir un rendement élevé en esters avec l'alcool méthylique ou l'alcool isopropylique qu'avec des alcools à longue chaîne, tels que les alcools butylique, amylique, eétylique, laurylique et stéarylique, car les alcools inférieurs et les esters lactiques correspondants sont solubles dans l'eau et ne forment pas de mélange azéotropique avec le benzène, le toluène, l'acétate d'éthyle. Il est, par conséquent, nécessaire de faire appel à des méthodes particulières pour éliminer l'eau au fur et à mesure de sa formation, ou bien on peut encore réduire la 
proportion d'eau par rapport à l'alcool dans le mélange réagissant, en utilisant un important excès d'alcool.

Voici d'ailleurs, d'après Smith et CLAвorn, la méthode générale que l'on peut employer pour la préparation des esters :

1. Esters à bas point d'ébullition, solubles dans l'eau :

5 molécules grammes, e'est-à-dire 1.090 grammes de lactate de calcium ou 10 molécules-grammes, c'est-à-dire 1.120 grammes de lactate de sodium, calculés en sec, sont dissous ou mélangés avee 50 molécules-grammes d'alcool méthylique (1.600 grammes), 2.300 grammes d'alcool éthylique ou 3.000 grammes d'alcool isopropylique. Une quantité équivalente d'acide sulfurique est ajoutée pour libérer l'acide lactique et précipiter le sulfate de calcium ou de sodium. Il faut ajouter également une quantité additionnelle de 20 grammes d'acide sulfurique comme catalyseur. On voit donc que, dans le mélange en réaction, les différents constituants sont dans les proportions suivantes : 1 partie d'acide lactique, 5 parties d'alcool, 0,02 partie d'acide sulfurique et 1 partie de sulfate de calcium ou 2 parties de sulfate de sodium, ces valeurs étant calculées en proportions moléculaires.

Ce mélange est alors porté à distillation au reflux pendant 4 à 8 heures, afin que l'éthérification soit complète. On le filtre et on recueille ainsi un liquide $\mathrm{F}_{1}$. Le résidu sur filtre est lavé avec de l'alcool frais, afin d'éliminer les esters résiduels. L'alcool de lavage représentant le liquide $\mathrm{F}_{2}$ est soumis à une distillation fractionnée pour récupérer l'ester qu'il contient, ou mis de côté pour servir à une préparation ultérieure. Le premier filtrat, c'est-à-dire le liquide $F_{1}$, est rapidement distillé à la pression atmosphérique, dans un flacon à col très court, sans être neutralisé de façon à éliminer l'excès d'alcool. On obtient ainsi une fraction alcoolique $\mathrm{D}_{\mathrm{l}}$. Lorsque la majeure partie de l'alcool a été éliminée de cette façon et que la température commence à s'élever, on poursuit l'opération dans le vide, et on réduit la pression jusqu'à ce que la majeure partie de l'ester ait été recueillie sous forme d'un distillat $\mathrm{D}_{2}$ qui contient encore un peu d'alcool, de l'eau et de l'ester.

La distillation doit être arrêtée avant qu'il ne se produise un commencement de carbonisation sous l'influence de l'excès d'acide sulfurique que contient le liquide. Le résidu $R_{1}$ qui reste dans le flacon renferme une certaine quantité d'ester, d'acide lactique n'ayant pas réagi, d'acide sulfurique, et de substances goudronneuses. En fabrication industrielle, où le problème ne s'impose pas de déterminer pour chaque opération le rendement exact, ce résidu est ajouté à une préparation ultérieure; si, au contraire, on veut déterminer le rendement, on le neutralise par de la chaux, on l'ex- 
trait à l'alcool et on récupère l'ester par distillation fractionnée de la solution alcoolique.

La fraction $\mathrm{D}_{1}$ est alors soumise à une distillation fractionnée, de façon à recueillir 3 parties : une partie dénommée fraction alcoolique A, une autre dénommée fraction alcoolique B et de l'eau contenant un peu d'ester. Il reste dans le flacon un résidu RC. Ce résidu $\mathrm{RC}$ est ajouté au produit $\mathrm{D}_{2}$ et soumis à une distillation fractionnée, sous pression réduite, en utilisant, à cet effet, un appareil particulièrement efficace. La première partie qui distille est ajoutée à la fraction B et sera soumise à une nouvelle distillation fractionnée. La seconde partie constitue l'ester purifié et enfin la partie restant dans l'appareil de distillation, dénommée résidu RD et qui contient une certaine quantité d'acide lactique, sera ajoutée à une préparation ultérieure.

Dans ces conditions, le rendement en ester raffiné, par rapport à l'acide lactique de départ, peut varier de 70 à $90 \%$. Ce rendement dépend de la quantité d'eau présente dans la matière première, de la technique employée pour la préparation, et de l'efficacité des appareils de distillation employés pour séparer l'excès d'alcool, l'eau et l'acide lactique non modifié de l'éther.

Parfois, le mélange éthérifié contient une certaine quantité de sulfate de calcium qu'il est difficile de séparer par filtration. Cette difficulté peut être surmontée en utilisant un plus grand excès d'alcool et une plus grande quantité d'acide sulfurique. On peut également augmenter le rendement de $10 \%$ et même plus en ester, en filtrant après éthérification, et en fractionnant le filtrat comme il a été décrit précédemment.

\section{Esters insolubles dans l'eau :}

Pour préparer les esters lactiques insolubles dans l'eau et ayant un point d'ébullition suffisamment bas pour qu'ils puissent être purifiés par distillation dans le vide, sans décomposition, on fait appel à un mode opératoire un peu différent. On mélange 5 molécules-grammes de lactate de calcium ou 10 molécules-grammes de lactate de sodium avec 1,5 à 2 molécules-grammes d'alcool (butylique, amylique, hexylique, octylique ou laurylique) et une quantité équivalente d'acide sulfurique augmentée de 10 grammes, cet excès devant servir de catalyseur. On ajoute également au mélange 50 grammes de benzène ou de toluène, de façon à former un mélange azéotropique, de façon à éliminer l'eau au fur et à mesure de sa. formation.

Le mélange doit être agité lentement, en même temps qu'on le chauffe à la température de reflux, et pendant toute la durée de la. réaction. Dans la colonne de reflux, on interpose un dispositif de 
PROPRIÉTÉS PHYSIQUĖS DES ESTERS LAGTIQUES

\begin{tabular}{|c|c|c|c|c|c|c|}
\hline \multirow{2}{*}{ Ester lactique } & \multicolumn{2}{|c|}{ Point d'ébullition } & \multirow{2}{*}{ Densité } & \multirow{2}{*}{$\begin{array}{c}\text { Indice } \\
\text { de } \\
\text { réfraction }\end{array}$} & \multicolumn{2}{|c|}{$\begin{array}{c}\text { Indice } \\
\text { de saponification }\end{array}$} \\
\hline & temp. ${ }^{\circ} \mathrm{C}$. & $\begin{array}{l}\text { pression } \\
\text { en mm. } \\
\text { mercure }\end{array}$ & & & calculé & mesuré \\
\hline Méthyle ....... & 144,8 & 760 & 1,0898 & 1,4132 & - & - \\
\hline Ethyle........ & 154,5 & 760 & 1,0308 & 1,4121 & 一 & - \\
\hline $\mathrm{N}$-propyle ..... & 86 & 40 & 0,996 & 1,4167 & 一 & - \\
\hline Isopropyle ..... & $166 / 8$ & 760 & 0,998 & 1,4082 & 一 & - \\
\hline N-butyle $\ldots \ldots$ & 185 & 760 & 0,973 & 1,4214 & 一 & - \\
\hline Isobutyle ...... & 96 & 40 & 0,971 & 1,4183 & 一 & - \\
\hline Butyle second. . & 180 & 760 & 0,974 & - & 一 & 一 \\
\hline $\mathrm{N}$-amyle $\ldots \ldots$ & 112 & 40 & 0,952 & 1,4254 & - & - \\
\hline Isoamyle...... & 82 & 7 & 0,9614 & 1,4240 & 350 & 353 \\
\hline N-hexyle...... & 75 & 2 & 0,9533 & 1,4290 & 322 & 322 \\
\hline 2-éthylbutyle .. & 104 & 12 & 0,9615 & 1,4307 & 322 & 321 \\
\hline 2-éthylhexyle .. & 112 & 3,6 & 0,9405 & 1,4358 & 277 & 278 \\
\hline Lauryle ...... & $150 / 3$ & 4 & 0,9108 & 1,4433 & 217 & 212 \\
\hline Phényléthyle... & 124 & 4 & 1,0979 & 1,5073 & 289 & 293 \\
\hline Glycol $\ldots \ldots \ldots$ & 140 & 10 & 1,1967 & 1,4452 & 419 & 413 \\
\hline Glycérine ..... & $175 / 80$ & 2 & - & - & - & - \\
\hline Benzyle ...... & 134 & 4 & 1,1355 & 1,5049 & - & - \\
\hline Stéaryle ...... & 180 & 2 & - & - & 一 & - \\
\hline $\begin{array}{l}\text { Ester acétoxy- } \\
\text { propionique }\end{array}$ & & & & & & + \\
\hline Méthyle ....... & 171,5 & 760 & 1,088 & 1,4111 & - & - \\
\hline Ethyle........ & 177 & 733 & 1,0458 & 1,4085 & 一 & - \\
\hline N-propyle .... & $195 / 6$ & 766 & 1,0163 & 1,4123 & - & - \\
\hline Isopropyle .... . & $182 / 3$ & 765 & 0,9920 & 1,4058 & - & - \\
\hline N-butyle ...... & $213 / 4$ & 767 & 1,0001 & 1,4147 & 一 & - \\
\hline Isobutyle ...... & 205 & 763 & 0,9952 & 1,4140 & 一 & 一 \\
\hline $\mathrm{N}$-amyle $\ldots \ldots$ & $226 / 7$ & 763 & 0,9822 & 1,4199 & - & - \\
\hline Isoamyle....... & $221 / 2$ & 763 & 0,9838 & 1,4190 & - & - \\
\hline N-hexyle...... & 135 & 17 & 0,9770 & 1,4232 & 519 & 519 \\
\hline 2-éthylbutyle .. & 127 & 14 & 0,9822 & 1,4245 & 519 & 522 \\
\hline 2-éthylhexyle .. & 145 & 13 & 0,9629 & 1,4298 & 460 & 462 \\
\hline Lauryle ...... & 165 & 4 & 0,9304 & 1,4373 & 373 & 370 \\
\hline Phényléthyle... & 139 & 4 & 1,0983 & 1,4896 & 475 & 476 \\
\hline Benzyle ...... & 145,8 & 4 & 1,1227 & 1,4874 & - & - \\
\hline
\end{tabular}


Betz-Holden, qui permet de séparer l'eau. Lorsque la quantité théorique d'eau qui doit se former au cours de la réaction, augmentée de celle qui pouvait exister dans les matières premières, s'est séparée, on peut admettre que l'estérification est complète. La réaction peut demander de 1 à 8 heures, suivant la vitesse de reflux. La purification par rectification de l'ester s'effectue comme il a été dit précédemment et on peut obtenir un rendement de 80 à $95 \%$ par rapport à l'acide lactique employé.

Pour la préparation d'esters lactiques purs dont le point d'ébullition est trop élevé pour permettre leur distillation dans le vide sans décomposition, il est indispensable d'utiliser comme point de départ de l'acide lactique purifié. Il n'est pas nécessaire non plus d'utiliser le catalyseur comme l'acide sulfurique. En utilisant un excès d'acide lactique, la totalité de l'alcool est estérifiée, et il suffit d'éliminer l'excès d'acide par lavage à l'eau. Les traces d'eau qui peuvent subsister dans l'ester sont éliminées par chauffage dans le vide.

Au cours de l'estérification, le produit a pu se colorer légèrement, on corrige ce défaut, en chauffant l'ester avec un charbon décolorant et en le filtrant. Le rendement que l'on obtient en produit parfaitement limpide est presque égal au rendement théorique.

3. Esters solubles dans l'eau à point d'ébullition élevé :

Pour la préparation des monolactates de glycol ou de glycérine, qui tous sont solubles dans l'eau et bouillent à une température élevée, il faut faire appel à une technique particulière. Il faut tout d'abord opérer en présence d'un important excès de ces alcools, afin d'éviter la formation ultérieure des lactides. On part d'une molécule-gramme, soit 90 grammes d'acide lactique pur à $100 \%$, de 5 molécules-grammes, soit 310 grammes de glycol ou 460 grammes de glycérine et 50 grammes de benzène. On chauffe au reflux jusqu'à ce que I'on ait séparé I molécule-gramme d'eau. On procède alors à une distillation fractionnée dans le vide, afin d'éliminer la totalité des glycols ou de la glycérine en excès. Le résidu encore chaud est traité par un charbon décolorant, puis filtré. On obtient ainsi un monolactate de haute qualité.

La pureté et le rendement en esters dépendent de la façon plus ou moins parfaite dont est séparé l'excès d'alcool. Les esters distillent en se décomposant légèrement et sous la pression de 10 millimètres de mercure à $140 / 145^{\circ}$ dans le cas du monolactate de glycol et de $180 / 190^{\circ}$ dans le cas du monolactate de glycérine. Il y a formation au cours de cette distillation d'une petite quantité de lactide et apparition de produits de coloration jaune et rappelant l'odeur de caramel. On peut d'ailleurs séparer le lactide sous forme cris- 
talline, en filtrant le distillat, après l'avoir abandonné toute une nuit à basse température.

Les dérivés acétoxy de ces esters lactiques se préparent en les: faisant réagir avec de l'anhydride acétique, suivant les méthodes: décrites par ClaboRN et SмIтH [10]. Le tableau ci-avant donne les principales caractéristiques physiques de ces esters.

\section{RÉFÉRENCES}

[1] Whitwier et Rogers. Ind. Eng. Chem., t. 23, p. 532, 1931.

[2] Ourve. Chem, and Met. Eng., t. 43, p. 480, 1936.

[3] Burton. Food Ind., t. 9, p. 634, 1937.

[4] Smith et Claborn. Ind. Eng. Chem., t. 32, p. 692, 1940.

[5] Wisticenus. Ann., t. 125, p. 58, 1863.

[6] Schreiner. Ann., t. 197, p. 12, 1879.

[7] Burns, Jones et Ritchie. Journ. Ohem. Soc., p. 400, 1935.

[8] AUger. C. R., t. 140, p. 938, 1905.

[9] Wislicenus. Loc. eit.

[10] Claborn et Smith. Journ. Am. Chem. Soc., t. 61, p. 2727, 1939.

\section{REVUE}

\section{CONSIDÉRATIONS SUR UNE CLASSIFICATION DES LAITS IMPROPRES A LA CONSOMMATION}

\section{par \\ A. HOUDINIÈRE}

Depuis longtemps de nombreux hygiénistes voudraient que soit établie une définition des laits impropres à la consommation humaine. Un tel souci répond à la légitime préoccupation de protéger la santé de nos semblables en éliminant par le jeu d'un contrôle hygiénique, les laits ainsi définis, de la production, de la préparation ou de la consommation (falsifications et fraudes mises à part).

Etant donné l'étendue du sujet, la question n'est pas simple. Essayons d'en dégager les limites et d'étudier les principes sur lesquels la classification et la définition des laits considérés doivent reposer.

\section{Valeur et interprétation.}

En matière de biologie plus que partout ailleurs, les classifications et définitions sont presque toujours très arbitraires et incomplètes. Elles le sont non seulement au regard du progrès continuel de nos connaissances, mais encore à celui de la relativité des choses, e'est-à-dire finalement de l'interprétation des faits. 